\title{
On An Optimization Problem with Hyperbolic Partial Differential Equations
}

\author{
M H FARAG \\ Mathematics and Statistics Department, Faculty of Science, Taif University \\ HAWIA (888) TAIF, SAUDI ARABIA
}

\begin{abstract}
In this paper an optimal control problem for a hyperbolic equation with a phase restriction is considered. The existence and the uniqueness of the solution of the problem are given. Using the penalty function method and introducing a conjugate problem, an expression of the gradient of the modified functional of the stated problem is found and the optimality condition is established.
\end{abstract}

Keywords: - Optimal control PDEs, Existence and Uniqueness theorem, Exterior penalty function, Hyperbolic Equations, Distributed parameter systems.

\section{INTRODUCTION AND STATEMENT OF THE PROBLEM}

Optimal control problems for hyperbolic equations have been investigated by Lions in his famous book [1]. Lions examined the problems in detail when the control function is at the right hand side and in the boundary condition of the hyperbolic problem. Furthermore, when the control is in the boundaries [2-4], in the coefficient [5-6], and at the right hand side of the equation [7, 8], there have been some control problem studies for different types of cost functional. As for the control of initial conditions, Lions mentioned the control of the initial velocity of the system in detail but stated briefly the control of initial status of the system solving the system in $L_{2}(\Omega)$.

Let $\Omega \in R_{n}$ is a bounded domain with smooth boundary $\Gamma ; x=\left(x_{1}, x_{2}, \cdots, x_{n}\right)$ an arbitrary point of the domain $\Omega, T>0$ is a given number $\quad 0 \leq t \leq T, Q_{T}=\Omega \times(0, T) \quad$ and $S_{T}=\Gamma \times(0, T)$. The notation of the functional spaces and their definitions are given in [9].

Throughout this paper, we adopt the following assumptions.

Assumption 1.1: $\Lambda=W_{P_{1}}^{1}(\Omega) \times W_{P_{2}}^{1}(\Omega) \times \cdots \times W_{P_{n}}^{1}(\Omega)$ be a space of controls, where $P_{i}, i=\overline{1, n}$ are given numbers, moreover $P_{i} n, i=\overline{1, n}$.

Assumption 1.2: $F(x, t) \in L_{2}\left(Q_{T}\right), \phi_{1} \in W_{2}^{1}(\Omega), \phi_{2} \in L_{2}(\Omega)$ are given functions.

Assumption 1.3: The function $G(x, t, y(x, t ; v))$ is given function, satisfying the Caratheodory conditions in the domain

$$
Q_{T} \times R \times U, U=\left\{v=\left(v_{1}(x), v_{2}(x), \cdots, v_{n}(x)\right) \in R_{n}: 0<\xi_{i}^{1} \leq v_{i}(x) \leq \xi_{i}^{2}(i=\overline{1, n})\right.
$$

is a domain of the values of admissible controls.

Assumption 1.4: Everywhere below positive constants are independent of the estimated quantities and admissible controls are denoted by $\Pi_{m}, m=1,2, \cdots$.

Assumption 1.5: There exists a function $B_{0}(x, t) \in L_{1}\left(Q_{T}\right)$ and a constant $\Pi_{1}>0$ such that $\forall(x, t) \in Q_{T}$ and $\forall y \in R, v \in U$ the inequality $|G(x, t, y, v)| \leq B_{0}(x, t)+\Pi_{1}|y|^{2} \quad$ holds true. 
Assumption 1.6: The function $G(x, t, y, v)$ has partial derivatives $\frac{\partial G}{\partial y}, \frac{\partial G}{\partial v_{i}}(i=1, \cdots, n)$ satisfying the Caratheodory conditions in the domain $Q_{T} \times R \times U$. Moreover, there exist constants $\Pi_{2}, \Pi_{3}>0$ and $d(x, t) \in L_{2}\left(Q_{T}\right), e(x, t) \in L_{1}\left(Q_{T}\right)$ such that $\stackrel{0}{\forall}(x, t) \in Q_{T}$ and $\forall y \in R, v \in U$ the inequalities

$$
\begin{gathered}
\left|\frac{\partial G(x, t, y, v)}{\partial y}\right| \leq d(x, t)+\Pi_{2}|y| \\
\left|\frac{\partial G(x, t, y, v)}{\partial v_{i}}\right| \leq e(x, t)+\Pi_{3}|y|^{2},(i=1, \cdots, n)
\end{gathered}
$$

hold true.

In this paper, we consider the following problem of minimizing the cost functional:

$$
f_{\alpha}(v)=\int_{Q_{T}} G(x, t, y(x, t ; v), v) d x d t
$$

under the following conditions:

and additional restriction

$$
\begin{gathered}
\frac{\partial^{2} y}{\partial t^{2}}-\sum_{i=1}^{n} \frac{\partial}{\partial x_{i}}\left(v_{i}(x) \frac{\partial y}{\partial x_{i}}\right)=F(x, t),(x, t) \in Q_{T} \\
y(x, 0)=\phi_{0}(x), \frac{\partial y(x, 0)}{\partial t}=\phi_{1}(x), x \in \Omega \\
\left.y\right|_{S_{T}}=0, x \in \Omega
\end{gathered}
$$

on the set of admissible controls

$$
\beta_{0} \leq y(x, t) \leq \beta_{1}
$$

$$
V_{i}=V_{1} \times V_{2} \times \cdots \times V_{n}
$$

and

$$
V_{i}=\left\{v_{i}(x) \in W_{P_{i}}^{1}(\Omega), 0<\xi_{i}^{1} \leq v_{i}(x) \leq \xi_{i}^{2},\left\|\frac{\partial v_{i}}{\partial x_{j}}\right\|_{L_{P_{i}}(\Omega)} \leq \overline{\xi_{i j}}, j=\overline{1, n}\right\}
$$

where $\xi_{i}^{1}, \xi_{i}^{2}, \overline{\xi_{i j}}(i, j=\overline{1, n})$ are given numbers, $v=\left(v_{1}(x), \cdots, v_{n}(x)\right)$ is a control and $y(x, t ; v)$ is the solution of the problem (2)-(4) corresponding to the control $v \in V$ and $\beta_{0}, \beta_{1}$ are given positive numbers.

Definition 1.1: The problem of finding a function $y=y(x, t ; v) \in W_{2}^{1,1}\left(Q_{T}\right)$ from conditions (2)-(4) for a given $v \in V$ be called the reduced problem.

Definition 1.2: A function $y=y(x, t ; v) \in W_{2}^{1,1}\left(Q_{T}\right)$ is said to be a generalized solution of the problem (2)-(4), if for all $\zeta=\zeta(x, t) \in W_{2}^{1,1}\left(Q_{T}\right)$ the equation 


$$
\begin{aligned}
\int_{Q_{T}}\left\{-\frac{\partial y(x, t)}{\partial t} \frac{\partial \zeta(x, t)}{\partial t}-\sum_{i=1}^{n} \frac{\partial y(x, t)}{\partial x_{i}} \frac{\partial \zeta(x, t)}{\partial x_{i}} d x d t\right\} \\
=\int_{Q_{T}} \zeta(x, t) F(x, t) d x d t+\int_{\Omega} \phi_{1}(x) \zeta(x, 0) d x
\end{aligned}
$$

is valid and $\zeta(x, T)=0$.

Using the above assumptions and the results of [9] the boundary value problem (2)-(4) has unique solution from $W_{2}^{1,1}\left(Q_{T}\right)$ for given $v \in V$ as follows:

Theorem 1.1: Suppose that $F(x, t) \in L_{2}\left(Q_{T}\right), v \in V_{i}, \phi_{1} \in L_{2}(\Omega)$ holds, then (2)-(4) a unique generalized solution from $W_{2}^{1,1}\left(Q_{T}\right)$ and the following estimate is valid for the solution:

$$
\|y(x, t)\|_{W_{2}^{1,1}\left(Q_{T}\right)} \leq \Pi_{4}\left\{\|F(x, t)\|_{L_{2}\left(Q_{T}\right)}+\left\|\phi_{1}(x)\right\|_{L_{2}(\Omega)}+\left\|\phi_{0}(x)\right\|_{W_{2}^{1}(\Omega)}\right\} .
$$

The constrained optimal control problem (1)-(5) is converted to an unconstrained control problem by adding a penalty function [10] to the cost functional (1), yielding the modified function

$$
\Psi_{\alpha, k}\left(v, r_{k}\right) \equiv \Psi(v)=f_{\alpha}(v)+P_{k}(v)
$$

where

$$
\begin{gathered}
\Phi^{1}(y)=\left\{\max \left[\beta_{0}-y(x, t ;) ; 0\right]\right\}^{2}, \Phi^{2}(y)=\left\{\max \left[y(x, t ;)-\beta_{2} ; 0\right]\right\}^{2} \\
P_{k}(v)=r_{k} \int_{0}^{l} \int_{0}^{T}\left[\Phi^{1}(y)+\Phi^{2}(y)\right] d x d t
\end{gathered}
$$

and $r_{k}>0, k=1,2, \cdots$ are positive numbers, $\lim _{k \rightarrow \infty} r_{k}=+\infty$.

We can state the following theorem of the existence and uniqueness of this optimal control in view of [11] as follows:

Theorem 1.2: Let the conditions in the statement of the problem (2)-(4) and the above assumptions be fulfilled. Then the set of optimal controls for the problem (1)-(4) $V^{*}=\left\{v^{*} \in V: f\left(v^{*}\right)=\inf \{v \in V\}\right\}$ is not empty and is weakly compact in $\Lambda$.

\section{OPTIMALITY CONDITIONS}

Now, we investigate the differential of the modified functional $\Psi_{\alpha, k}\left(v, r_{k}\right)$. For this purpose, we consider the following adjoint boundary value problem

$$
\begin{aligned}
\frac{\partial^{2} \Theta}{\partial t^{2}}-\sum_{i=1}^{n} \frac{\partial}{\partial x_{i}}\left(v_{i}(x) \frac{\partial \Theta}{\partial x_{i}}\right)=-\frac{\partial G(x, t, y, v)}{\partial y} & \\
& -r_{k}\left[\Phi^{1}(y)+\Phi^{2}(y)\right], \quad(x, t) \in Q_{T}
\end{aligned}
$$




$$
\begin{gathered}
\Theta(x, T)=0, \frac{\partial \Theta(x, T)}{\partial t}=0, x \in \Omega \\
\left.\Theta\right|_{S_{T}}=0, x \in \Omega
\end{gathered}
$$

Here, the function $y \equiv y(x, t ; v)$ is the solution of problem (1)-(3) for $v \in V$.

The generalized solution $\Theta(x, t)$ of (11)-(13) is a function which belongs to $W_{2}^{1,1}\left(Q_{T}\right)$ equal zero at $t=0$ and satisfies the following integral equation

$$
\begin{gathered}
\int_{Q_{T}}\left[-\frac{\partial \Theta}{\partial t} \frac{\partial \lambda}{\partial t}+\sum_{i=1}^{n} v_{i}(x) \frac{\partial \lambda}{\partial x_{i}} \frac{\partial \Theta}{\partial x_{i}}\right] d x d t \\
=-\int_{Q_{T}} \frac{\partial G(x, t, y, v)}{\partial y} \lambda(x, t) d x d t+r_{k} \int_{Q_{T}}\left[\Phi^{1}(y)+\Phi^{2}(y)\right] \lambda(x, t) d x d t
\end{gathered}
$$

where $\forall \lambda=\lambda(x, t) \in W_{2}^{1,1}\left(Q_{T}\right)$ and $\lambda(x, 0)=0$.

It follows from the results of [9, p.209-215] that under the adapted assumptions for each fixed $v \in V$ the boundary value problem (11)-(13) has a unique solution from $W_{2}^{1,1}\left(Q_{T}\right)$ and the estimation

$$
\|\Theta\|_{W_{2}^{1,1}\left(Q_{T}\right)} \leq \Pi_{5}\left\|\frac{\partial G(x, t, y, v)}{\partial y}\right\|_{L_{2}\left(Q_{T}\right)}
$$

is valid.

Theorem 2.1: Let the conditions Theorem 1.1, assumption 1.5 and for the solution of the problem (2)-(4) $y^{*} \equiv y\left(x, t ; v^{*}\right)$, the conditions $\frac{\partial^{2} y^{*}}{\partial x_{i}^{2}} \in L_{2}\left(Q_{T}\right), i=\overline{1, n}$ be fulfilled. Then the functional $\Psi_{\alpha, k}\left(v, r_{k}\right)$ is continuously differentiable by Frechet in $V$ and its differential at the point $v \in V$ at the increment $\Delta v=\left(\Delta v_{1}, \Delta v_{2}, \cdots, \Delta v_{n}\right) \in \Lambda$ is determined by the expression

$$
\prec \Psi^{\prime}\left(v^{*}\right), \Delta v \succ=\int_{Q_{T}} \sum_{i=1}^{n}\left\{-\frac{\partial \Theta^{*}}{\partial x_{i}} \frac{\partial y^{*}}{\partial x_{i}}+\frac{\partial G(x, t, y, v)}{\partial v_{i}}\right\} \Delta v_{i} d x d t
$$

where $\Theta^{*}=\Theta\left(x, t ; v^{*}\right)$ is a solution of the problem (11)-(13) for $v=v^{*}$ and $\prec \Psi^{\prime}\left(v^{*}\right), \Delta v \succ$ means the value of $\Psi^{\prime}\left(v^{*}\right)$ on the element $\Delta v$.

Proof: Now, using the Lagrange formula for the increment of the modified functional $\Psi_{\alpha, k}\left(v, r_{k}\right)$ we obtain the formula 


$$
\begin{aligned}
\Delta \Psi_{v}\left(v^{*}\right)= & \Psi_{v}\left(v^{*}+\Delta v\right)-\Psi_{v}\left(v^{*}\right) \\
= & \int_{Q_{T}}\left[\frac{\partial G(x, t ; v)}{\partial y} \Delta y+\sum_{i=1}^{n} \frac{\partial G(x, t ; v)}{\partial v_{i}} \Delta v_{i}\right] d x d t \\
& +r_{k} \int_{0}^{l} \int_{0}^{T}\left[\frac{\partial \Phi^{1}(y)}{\partial y}+\frac{\partial \Phi^{2}(y)}{\partial y}\right] \Delta y(x, t) d x d t+\mathfrak{R}_{1}
\end{aligned}
$$

where

$$
\begin{aligned}
\mathfrak{R}_{1}=\int_{Q_{T}}\left\{\left[\frac{\partial G\left(\sigma_{\rho 1}\right)}{\partial y}-\frac{\partial G}{\partial y}\right] \Delta y+\sum_{i=1}^{n}\left[\frac{\partial G\left(\sigma_{\rho 1}\right)}{\partial v_{i}}-\frac{\partial G}{\partial v_{i}}\right] \Delta v_{i} d x d t\right. \\
\quad+r_{k} \int_{Q_{T}}[\Delta y(x, t)]^{2} d x d t
\end{aligned}
$$

and $\sigma_{\rho 1}=\left(x, t, y^{*}+\rho 1 \Delta y, v^{*}+\rho 1 \Delta v\right), 0 \leq \rho 1 \leq 1, \rho 1 \in[0,1]$ is a number.

Let $\Delta v=\left(\Delta v_{1}, \Delta v_{2}, \cdots, \Delta v_{n}\right) \in \Lambda$ be an arbitrary increment of the control $v^{*} \in V$ such that $v^{*}+\Delta v \in V$. Using the Lagrange's formula finite increments one can obtain that the function $\Delta y=y\left(x, t, v^{*}+\Delta v\right)-y\left(x, t, v^{*}\right)$ is a solution from the class $W_{2}^{1,1}\left(Q_{T}\right)$ of the following boundary value problem:

$$
\begin{gathered}
\frac{\partial^{2} \Delta y}{\partial t^{2}}+\sum_{i=1}^{n} \frac{\partial}{\partial x_{i}}\left(\left\{v_{i}^{*}(x)+\Delta v_{i}\right\} \frac{\partial \Delta y}{\partial x_{i}}\right)=\sum_{i=1}^{n} \frac{\partial}{\partial x_{i}}\left(\Delta v_{i} \frac{\partial \Delta y}{\partial x_{i}}\right),(x, t) \in Q_{T} \\
\Delta y(x, T)=0, \frac{\partial \Delta y(x, 0)}{\partial t}=0, x \in \Omega \\
\left.\Delta y\right|_{S_{T}}=0, x \in \Omega
\end{gathered}
$$

Then under the given conditions for the solution of the problem (19)-(21) the estimation

$$
\begin{gathered}
\|\Delta y(x, t)\|_{W_{2}^{1,1}\left(Q_{T}\right)} \leq \Pi_{6} \lambda \\
\lambda=\left\{\sum_{i=1}^{n}\left\|\Delta v_{i} \frac{\partial^{2} \Delta y^{*}}{\partial x_{i}^{2}}\right\|_{L_{2}\left(Q_{T}\right)}^{2}+\sum_{i=1}^{n}\left\|\frac{\partial \Delta v_{i}}{\partial x_{i}} \frac{\partial y^{*}}{\partial x_{i}}\right\|_{L_{2}\left(Q_{T}\right)}^{2}\right\}
\end{gathered}
$$

is valid.

Using the boundedness of the imbedding's $\quad W_{P_{i}}^{1}(\Omega) \rightarrow C(\bar{\Omega})$ for $P_{i}>n, i=\overline{1, n}$, the above assumption 1.5, the condition of the Theorem, estimation (9) and estimating the right side of (22) we establish that

$$
\|\Delta y\|_{W_{2}^{1,1}\left(Q_{T}\right)} \leq \Pi_{7}\left\|\Delta v_{i}\right\|_{\Lambda}
$$

The solution of the problem (19)-(21) satisfies the equality 


$$
\begin{aligned}
\int_{Q_{T}}\left\{\frac{\partial \Delta y}{\partial t} \frac{\partial \Theta^{*}}{\partial t}\right. & \left.+\sum_{i=1}^{n}\left(v_{i}^{*}(x)+\Delta v_{i}\right) \frac{\partial \Theta^{*}}{\partial x_{i}} \frac{\partial \Delta y}{\partial x_{i}}\right\} d x d t \\
& =-\int_{Q_{T}} \sum_{i=1}^{n} \frac{\partial y^{*}}{\partial x_{i}} \frac{\partial \Theta^{*}}{\partial x_{i}} \Delta v_{i} d x d t
\end{aligned}
$$

If in (14) for $v^{*} \in V$ we put $\lambda=\Delta y$ and subtract the obtained equality from (24), we can obtain

$$
\begin{gathered}
\int_{Q_{T}}\left\{\frac{\partial G}{\partial y}+r_{k}\left[\frac{\partial \Phi^{1}}{\partial y}+\frac{\partial \Phi^{2}}{\partial y}\right]\right\} \Delta y(x, t ; v) d x d t \\
=\int_{Q_{T}} \sum_{i=1}^{n} \frac{\partial y^{*}}{\partial x_{i}} \frac{\partial \Theta^{*}}{\partial x_{i}} \Delta v_{i} d x d t+\mathfrak{R}_{2}
\end{gathered}
$$

where $\mathfrak{R}_{2}=\int_{Q_{T}} \sum_{i=1}^{n} \frac{\partial \Delta y}{\partial x_{i}} \frac{\partial \Theta^{*}}{\partial x_{i}} \Delta v_{i} d x d t$

In view of this, we write relation (17) in the form

$$
\Delta \Psi_{v}\left(v^{*}\right)=\int_{Q_{T}} \sum_{i=1}^{n}\left[\frac{\partial y^{*}}{\partial x_{i}} \frac{\partial \Theta^{*}}{\partial x_{i}}+\frac{\partial G(x, t, y ; v)}{\partial v_{i}}\right] \Delta v_{i} d x d t+\mathfrak{R}_{1}+\mathfrak{R}_{2}
$$

Using the estimations (22), (23) we can estimate the reminder terms $\mathfrak{R}_{1}, \mathfrak{R}_{2}$ as follows:

$$
\begin{gathered}
\left|\Re_{1}\right| \leq \Pi_{5}\left[\left\|\frac{\partial G\left(\sigma_{\rho 1}\right)}{\partial y}-\frac{\partial G}{\partial y}\right\|_{L_{2}(\Omega)}+\sum_{i=1}^{n}\left\|\frac{\partial G\left(\sigma_{\rho 1}\right)}{\partial v_{i}}-\frac{\partial G}{\partial v_{i}}\right\|_{L_{2}(\Omega)}\right]\|\Delta v\|_{\Lambda}+r_{k}\|\Delta v\|_{\Lambda} \\
\left|\Re_{2}\right|=\left|\int_{Q_{T}} \sum_{i=1}^{n} \frac{\partial \Delta y}{\partial x_{i}} \frac{\partial \Theta^{*}}{\partial x_{i}} \Delta v_{i} d x d t\right| \\
\leq \Pi_{8}\left[\sum_{i=1}^{n}\left\|\frac{\partial \Delta y}{\partial x_{i}}\right\| \times\left\|\frac{\partial \Theta^{*}}{\partial x_{i}}\right\|\right] \leq \Pi_{9}\|\Delta v\|_{\Lambda}
\end{gathered}
$$

It follows from the above two inequalities that $\mathfrak{R}_{k}=O\left(\|\Delta v\|_{\Lambda}\right), k=1,2$ is valid for the reminder term $\mathfrak{R}=\mathfrak{R}_{1}+\mathfrak{R}_{2}$. By taking into account the estimate of $\mathfrak{R}$ in (26), we find that the functional $\Psi_{\alpha, k}\left(v, r_{k}\right)$ is Fr'echet differentiable on $V$ and its differential is given by (16). The proof of Theorem 2.1 is complete.

The following assertion provides a necessary optimality condition for the problem (2)-(4),(6).

Theorem 2.2: Let the assumptions of the problem be fulfilled. Then for the optimality of control $v^{*}=\left(v_{1}, v_{2}, \cdots, v_{n}\right) \in V$ in the problem (1)-(4),(7) it is necessary that the inequality 


$$
\int_{Q_{T}}\left\{\sum_{i=1}^{n}\left[\frac{\partial y^{*}}{\partial x_{i}} \frac{\partial \Theta^{*}}{\partial x_{i}}+\frac{\partial G(x, t, y ; v)}{\partial x_{i}}\right] \times\left(v_{i}(x)-v_{i}^{*}(x)\right)\right\} d x d t \geq 0,
$$

be fulfilled for an arbitrary $v^{*} \in V$, where $y^{*}=y^{*}\left(x, t, v^{*}\right)$ and $\Theta^{*}=\Theta\left(x, t, v^{*}\right)$ are the solutions of the problems (1)-(4),(7) and (11)-(13) for $v \in V$.

Proof: The set $V$ is convex in $\Lambda$. Besides, by Theorem 2.1 the functional $\Psi_{\alpha, k}\left(v, r_{k}\right)$ is continuously differentiable by Freshet in $V$. Then by Theorem 5 from [11, p. 524] it is necessary that the inequality $\prec \frac{\partial \Psi\left(v^{*}\right)}{\partial v}, v-v^{*} \succ \geq 0$ be fulfilled for all $v \in V$ on the element $v^{*} \in V^{*}$. The validity of the inequality (29) follows from this and (16). Theorem 2.2 is proved.

\section{CONCLUSION}

In this paper, we prove the existence and the uniqueness of an optimal control problem for a hyperbolic equation with a phase restriction. Applying the penalty function method and introducing a conjugate problem, an expression of the gradient of the modified functional of the stated problem is found. Finally, we prove the optimality conditions.

\section{REFERENCES}

[1] J.-L Lions, Optimal control by systems described by partial differential equations (Mir, Moscow, SSSR, 1972).

[2] H. F. Guliyev and K. SH. Jabarova, On an optimal control problem for weakly nonlinear hyperbolic equations, Acta Math. Hungar, 12(5), 2011, 24-34.

[3] S. Yesim and S. Murat, On the regularized Solutions of optimal control problem in a hyperbolic system, Abstract and Applied Analysis, Article ID 156541, 2012, 1-12.

[4] Z.S. Wu, K.L. Teo, A conditional gradient method for an optimal control problem involving a class of nonlinear second-order hyperbolic partial differential equations, Journal of Mathematical Analysis and Applications, (91) 2, 1983, 376-393.

[5] F. Periago, Optimal shape and position of the support for the internal exact control of a string, Systems and Control Letters, 58(2), 2009, 136-140.

[6] T. M. Mustafayeva, On an optimal control problem for a hyperbolic equation with phase restriction, Proc. of IMM of NAS of Azerbaijan. XXXVI (XLIV), 2012, 117-124.

[7] Serovskiy S. Ya., Optimization of nonlinear hyperbolic equations at nonavailability of uniqueness theorem of the boundary value problem solution, Izv. Vuzov. Matematika, (1), 2009, 76-83.

[8] F. H. GULIYEV, T. M. Mustafayeva, Optimal stabilization far a weak nonlinear hyperbolic with phase restriction, Transactions of NAS of Azerbaijan, XXXII (1), 2012, 53-60.

[9] O. A. Ladyzhenskaya, Boundary value problems of mathematical physics (Nauka, Moscow, Russian, 1973).

[10] Joghataie and M. Takalloozadeh, Improving Penalty Functions for Structural Optimization (Sharif University of Technology, Tehran, Iran, 2009).

[11] F. P. Vasilev, Methods of Optimization (Moscow, Factorial press, Russian, 2002).

\begin{tabular}{|l|l|}
\hline & $\begin{array}{l}\text { Prof. Dr. Mahmoud Hashem Farag } \\
\text { Professor of Mathematics (Numerical Analysis ,Numerical Optimization, Optimal } \\
\text { control PDEs), Mathematics Department - Faculty of Science - Minia University - } \\
\text { Minia - Egypt OR Mathematics and Statistics Department, Faculty of Science, } \\
\text { TAIF University, HAWIA (888) TAIF, SAUDI ARABIA. }\end{array}$ \\
\hline
\end{tabular}

